\title{
Search for nucleon decay via modes favored by supersymmetric grand unification models in Super-Kamiokande-I
}

K.Kobayashi, ${ }^{1}$ M.Earl,, , 田 Y.Ashie, ${ }^{3}$ J.Hosaka, ${ }^{3}$ K.Ishihara, ${ }^{3}$ Y.Itow, ${ }^{3}$ J.Kameda,${ }^{3}$ Y.Koshio, ${ }^{3}$ A.Minamino, ${ }^{3}$ C.Mitsuda, ${ }^{3}$ M.Miura, ${ }^{3}$ S.Moriyama, ${ }^{3}$ M.Nakahata, ${ }^{3}$ T.Namba,${ }^{3}$ R.Nambu, ${ }^{3}$ Y.Obayashi,,${ }^{3}$ M.Shiozawa, ${ }^{3}$ Y.Suzuki, ${ }^{3}$ Y.Takeuchi, ${ }^{3}$ K.Taki,${ }^{3}$ S.Yamada, ${ }^{3}$ M.Ishitsuka,${ }^{4}$ T.Kajita,${ }^{4}$ K.Kaneyuki, ${ }^{4}$ S.Nakayama,${ }^{4}$ A.Okada, ${ }^{4}$ K.Okumura,${ }^{4}$ T.Ooyabu,${ }^{4}$ C.Saji, ${ }^{4}$ Y.Takenaga, ${ }^{4}$ S.Desai, ${ }^{2}$ E.Kearns, ${ }^{2}$ S.Likhoded, ${ }^{2}$ J.L.Stone, ${ }^{2}$ L.R.Sulak, ${ }^{2}$ W.Wang, ${ }^{2}$ M.Goldhaber,${ }^{5}$ D.Casper,${ }^{6}$ J.P.Cravens, ${ }^{6}$ W.Gajewski, ${ }^{6}$ W.R.Kropp,${ }^{6}$ D.W.Liu,${ }^{6}$ S.Mine, ${ }^{6}$ M.B.Smy,${ }^{6}$ H.W.Sobel,${ }^{6}$ C.W.Sterner,${ }^{6}$ M.R.Vagins, ${ }^{6}$ K.S.Ganezer, ${ }^{7}$ J.E.Hill,,${ }^{7}$ W.E.Keig, ${ }^{7}$ J.S.Jang, ${ }^{8}$ J.Y.Kim,${ }^{8}$ I.T.Lim,${ }^{8}$ K.Scholberg, ${ }^{9}$ C.W.Walter,${ }^{9}$ R.W.Ellsworth,,${ }^{10}$ S.Tasaka, ${ }^{11}$ G.Guillian, ${ }^{12}$ A.Kibayashi, ${ }^{12}$ J.G.Learned, ${ }^{12}$ S.Matsuno, ${ }^{12}$ D.Takemori, ${ }^{12}$ M.D.Messier, ${ }^{13}$ Y.Hayato, ${ }^{14}$ A.K.Ichikawa, ${ }^{14}$ T.Ishida, ${ }^{14}$ T.Ishii, ${ }^{14}$ T.Iwashita, ${ }^{14}$ T.Kobayashi, ${ }^{14}$ T.Maruyama, ${ }^{14, \text { U K.Nakamura }}{ }^{14}$ K.Nitta, ${ }^{14}$ Y.Oyama, ${ }^{14}$ M.Sakuda, ${ }^{14}$ 团 Y.Totsuka, ${ }^{14}$ A.T.Suzuki, ${ }^{15}$ M.Hasegawa, ${ }^{16}$ K.Hayashi, ${ }^{16}$ I.Kato, ${ }^{16}$ H.Maesaka, ${ }^{16}$ T.Morita, ${ }^{16}$ T.Nakadaira, ${ }^{16}$ T.Nakaya, ${ }^{16}$ K.Nishikawa, ${ }^{16}$ T.Sasaki, ${ }^{16}$ S.Ueda, ${ }^{16}$ S.Yamamoto, ${ }^{16}$ M.Yokoyama, ${ }^{16}$ T.J.Haines,${ }^{17,6}$ S.Dazeley ${ }^{18}$ S.Hatakeyama, ${ }^{18}$ R.Svoboda, ${ }^{18}$ E.Blaufuss, ${ }^{19}$ J.A.Goodman, ${ }^{19}$ G.W.Sullivan, ${ }^{19}$ D.Turcan, ${ }^{19}$ A.Habig, ${ }^{20}$ Y.Fukuda, ${ }^{21}$ C.K.Jung, ${ }^{1}$ T.Kato,${ }^{1}$ M.Malek, ${ }^{1}$ C.Mauger,${ }^{1}$ C.McGrew,${ }^{1}$ A.Sarrat,${ }^{1}$ E.Sharkey, ${ }^{1}$ C.Yanagisawa, ${ }^{1}$ T.Toshito, ${ }^{22}$ K.Miyano, ${ }^{23}$ N.Tamura, ${ }^{23}$ J.Ishii, ${ }^{24}$ Y.Kuno, ${ }^{24}$ M.Yoshida, ${ }^{24}$ S.B.Kim, ${ }^{25}$ J.Yoo, ${ }^{25}$ H.Okazawa, ${ }^{26}$ T.Ishizuka, ${ }^{27}$ Y.Choi, ${ }^{28}$ H.K.Seo,${ }^{28}$ Y.Gando, ${ }^{29}$ T.Hasegawa, ${ }^{29}$ K.Inoue, ${ }^{29}$ J.Shirai, ${ }^{29}$ A.Suzuki, ${ }^{29}$ M.Koshiba, ${ }^{30}$ Y.Nakajima, ${ }^{31}$ K.Nishijima, ${ }^{31}$ T.Harada ${ }^{32}$ H.Ishino, ${ }^{32}$ Y.Watanabe, ${ }^{32}$ D.Kielczewska, ${ }^{33,6}$ J.Zalipska, ${ }^{33}$ H.G.Berns,${ }^{34}$ R.Gran, ${ }^{34}$ K.K.Shiraishi, ${ }^{34}$ A.Stachyra, ${ }^{34}$ K.Washburn, ${ }^{34}$ and R.J.Wilkes ${ }^{34}$

(The Super-Kamiokande Collaboration)

${ }^{1}$ Department of Physics and Astronomy, State University of New York, Stony Brook, NY 11794-3800, USA

${ }^{2}$ Department of Physics, Boston University, Boston, MA 02215, USA

${ }^{3}$ Kamioka Observatory, Institute for Cosmic Ray Research, University of Tokyo, Kamioka, Gifu, 506-1205, Japan

${ }^{4}$ Research Center for Cosmic Neutrinos, Institute for Cosmic Ray

Research, University of Tokyo, Kashiwa, Chiba 277-8582, Japan

${ }^{5}$ Physics Department, Brookhaven National Laboratory, Upton, NY 11973, USA

${ }^{6}$ Department of Physics and Astronomy, University of California, Irvine, Irvine, CA 92697-4575, USA

${ }^{7}$ Department of Physics, California State University, Dominguez Hills, Carson, CA 90747, USA

${ }^{8}$ Department of Physics, Chonnam National University, Kwangju 500-757, Korea

${ }^{9}$ Department of Physics, Duke University, Durham, NC 27708 USA

${ }^{10}$ Department of Physics, George Mason University, Fairfax, VA 22030, USA

${ }^{11}$ Department of Physics, Gifu University, Gifu, Gifu 501-1193, Japan

${ }^{12}$ Department of Physics and Astronomy, University of Hawaii, Honolulu, HI 96822, USA

${ }^{13}$ Department of Physics, Indiana University, Bloomington, IN 47405-7105, USA

${ }^{14}$ High Energy Accelerator Research Organization (KEK), Tsukuba, Ibaraki 305-0801, Japan

${ }^{15}$ Department of Physics, Kobe University, Kobe, Hyogo 657-8501, Japan

${ }^{16}$ Department of Physics, Kyoto University, Kyoto 606-8502, Japan

${ }^{17}$ Physics Division, P-23, Los Alamos National Laboratory, Los Alamos, NM 87544, USA

${ }^{18}$ Department of Physics and Astronomy, Louisiana State University, Baton Rouge, LA 70803, USA

${ }^{19}$ Department of Physics, University of Maryland, College Park, MD 20742, USA

${ }^{20}$ Department of Physics, University of Minnesota, Duluth, MN 55812-2496, USA

${ }^{21}$ Department of Physics, Miyagi University of Education, Sendai, Miyagi 980-0845, Japan

${ }^{22}$ Department of Physics, Nagoya University, Nagoya, Aichi 464-8602, Japan

${ }^{23}$ Department of Physics, Niigata University, Niigata, Niigata 950-2181, Japan

${ }^{24}$ Department of Physics, Osaka University, Toyonaka, Osaka 560-0043, Japan

${ }^{25}$ Department of Physics, Seoul National University, Seoul 151-742, Korea

${ }^{26}$ International and Cultural Studies, Shizuoka Seika College, Yaizu, Shizuoka 425-8611, Japan

${ }^{27}$ Department of Systems Engineering, Shizuoka University, Hamamatsu, Shizuoka 432-8561, Japan

${ }^{28}$ Department of Physics, Sungkyunkwan University, Suwon 440-746, Korea

${ }^{29}$ Research Center for Neutrino Science, Tohoku University, Sendai, Miyagi 980-8578, Japan

${ }^{30}$ University of Tokyo, Tokyo 113-0033, Japan

${ }^{31}$ Department of Physics, Tokai University, Hiratsuka, Kanagawa 259-1292, Japan

${ }^{32}$ Department of Physics, Tokyo Institute for Technology, Meguro, Tokyo 152-8551, Japan

${ }^{33}$ Institute of Experimental Physics, Warsaw University, 00-681 Warsaw, Poland

${ }^{34}$ Department of Physics, University of Washington, Seattle, WA 98195-1560, USA

(Dated: September 19, 2018)

We report the results for nucleon decay searches via modes favored by supersymmetric grand unified models in Super-Kamiokande. Using 1489 days of full Super-Kamiokande-I data, we searched 
for $p \rightarrow \bar{\nu} K^{+}, n \rightarrow \bar{\nu} K^{0}, p \rightarrow \mu^{+} K^{0}$ and $p \rightarrow e^{+} K^{0}$ modes. We found no evidence for nucleon decay in any of these modes. We set lower limits of partial nucleon lifetime $2.3 \times 10^{33}, 1.3 \times 10^{32}, 1.3 \times 10^{33}$ and $1.0 \times 10^{33}$ years at $90 \%$ confidence level for $p \rightarrow \bar{\nu} K^{+}, n \rightarrow \bar{\nu} K^{0}, p \rightarrow \mu^{+} K^{0}$ and $p \rightarrow e^{+} K^{0}$ modes, respectively. These results give a strong constraint on supersymmetric grand unification models.

PACS numbers: Valid PACS appear here

\section{INTRODUCTION}

Grand Unified Theories (GUTs) 1, 2] seek to unify the strong and electroweak forces. They are motivated by the apparent merging of the coupling constants of the strong, weak, and electromagnetic forces at a large energy scale $\left(\sim 10^{16} \mathrm{GeV}\right)$ when low energy measurements are extrapolated. One of the generic predictions of GUTs is the instability of the proton, as well as neutrons bound inside the nucleus. The experimental observation of nucleon decay would provide a strong evidence of GUTs.

In GUTs, nucleon decay can proceed via an exchange of a massive boson between two quarks in a proton or in a bound neutron. In this reaction, one quark transforms into a lepton and another into an anti-quark which binds with a spectator quark creating a meson. The favored decay mode in the prototypical GUT [2] based on an $S U(5)$ symmetry ("minimal $S U(5)$ ") is $p \rightarrow e^{+} \pi^{0}$. For this decay, the proton lifetime scales as $\sim M_{X}^{4}$, where $M_{X}$ is the mass of the heavy vector gauge boson. In minimal $S U(5), M_{X}$ is on the order of the coupling unification at $10^{15} \mathrm{GeV} / c^{2}$, yielding a predicted proton lifetime of $\tau / B\left(p \rightarrow e^{+} \pi^{0}\right) \sim 10^{29 \pm 2}$ years. The first generation large water Cherenkov detector experiments 3, 4], motivated by this prediction, observed no evidence of proton decay in this mode and ruled out the model. Also, the recent result by the Super-Kamiokande experiment extended the previous results [5]. It turns out that this contradiction of $S U(5)$ with the experimental proton decay limit can be resolved by incorporating supersymmetry (SUSY) in the theories.

Supersymmetry postulates that, for every SM particle, there is a corresponding "superpartner" with spin differing by $1 / 2$ unit from the SM particle [6]. The additional particles stabilize the renormalization of the Higgs boson and address the so-called "hierarchy problem". When one incorporates the superpartners into the calculation of the running of the coupling constants, the convergence of the coupling constants occurs at an unification scale about one order of magnitude larger than that of minimal $S U(5)$. Since the proton decay rate via $p \rightarrow e^{+} \pi^{0}$ scales as $M_{X}^{-4}$, this leads to a suppression of about four orders

\footnotetext{
* Present address: University of Maryland School of Medicine, Baltimore, MD 21201, USA

${ }^{\dagger}$ Present address: Department of Physics, Univ. of Tsukuba, Tsukuba, Ibaraki 305 8577, Japan

${ }^{\ddagger}$ Present address: Department of Physics, Okayama University, Okayama 700-8530, Japan
}

of magnitude in the rate, consistent with experimental non-observation of $p \rightarrow e^{+} \pi^{0}$. Furthermore, while in the minimal $S U(5)$ model, the three coupling constants do not quite meet at a single point within three standard deviations, they meet together at a single point in the minimal SUSY $S U(5)$ model [7].

However, in many SUSY GUT models, other dominant nucleon decay modes occur via dimension five operator interactions with the exchange of a heavy supersymmetric color triplet Higgsino [8]. These interactions suppress transitions from one quark family in the initial state to the same family in the final state. Since the only second or third generation quark which is kinematically allowed is the strange quark, an anti-strange quark typically appears in the final state for these interactions. The antistrange quark binds with a spectator quark to form a $K$ meson in the final state. Thus, SUSY GUTs favor nucleon decays in $p \rightarrow \bar{\nu} K^{+}$and $n \rightarrow \bar{\nu} K^{0}$ modes. The predictions for the nucleon lifetime in SUSY GUT models, however, varies widely, and may even be suppressed, since there are many new free parameters introduced because of the supersymmetry breaking.

In the minimal SUSY $S U(5)$ GUTs, the partial proton lifetime is estimated to be $\tau / B\left(p \rightarrow \bar{\nu} K^{+}\right) \leq 2.9 \times 10^{30}$ years $[9]$. Other models have been proposed beyond those based on $S U(5)$. In particular, models based on $S O(10)$ symmetry have become popular in light evidence for neutrino mass [10], which they naturally accommodate. An important property of the $S O(10)$ symmetry is that there is a heavy right-handed neutrino in a multiplet containing the matter fields. In addition, all matter fields of one generation can be contained in a single multiplet, in contrast to theories based on $S U(5)$ where they must be broken into two separate representations.

One class of models 11], predicts the partial proton lifetime for $p \rightarrow \bar{\nu} K^{+}$mode to be less than $10^{34}$ years, which is within the observable range of SuperKamiokande. In addition, the same mechanism which gives mass to the neutrinos provides a new set of dimension five operators through which the proton can decay. A consequence of this is that the prediction for $p \rightarrow \mu^{+} K^{0}$ decay rate is within a factor of 10 of the $p \rightarrow \bar{\nu} K^{+}$decay rate.

These decay modes, favored by SUSY GUT models, have been searched for in water Cherenkov detector $\underline{3}$, [4, 29 and iron calorimeter 12, 13] experiments. The best limits on the partial nucleon lifetime via $p \rightarrow \bar{\nu} K^{+}$, $n \rightarrow \bar{\nu} K^{0}, p \rightarrow \mu^{+} K^{0}$, and $p \rightarrow e^{+} K^{0}$ are $6.7 \times 10^{32}$ years [29], $8.6 \times 10^{31}$ years [4], $1.2 \times 10^{31}$ years [3, 4, 13], and $1.5 \times 10^{31}$ years [4], respectively. 


\section{SUPER-KAMIOKANDE DETECTOR}

The Super-Kamiokande detector [14] is a 50 kton water Cherenkov detector located at the Kamioka Observatory of Institute for Cosmic Ray Research in the Kamioka mine. It lies 1,000 $\mathrm{m}$ underneath the top of Mt. Ikenoyama, (i.e. 2,700 $\mathrm{m}$ water equivalent underground), resulting in a cosmic ray muon rate of $2.2 \mathrm{~Hz}$, a reduction of $10^{-5}$ compared to the rate at the surface. The detector is optically separated into two regions, the inner and outer detectors (ID and OD). The ID of the SuperKamiokande-I detector, which operated from April 1996 to July 2001, was instrumented with 11,146 50-cm diameter inward facing photomultiplier tubes (PMTs) which provide $40 \%$ photocathode coverage. This photocathode coverage makes it possible to detect low energy electrons down to $\sim 5 \mathrm{MeV}$. The ID volume is the sensitive region for nucleon decay searches and has a total fiducial volume of $22.5 \mathrm{kton}$, defined as a cylindrical volume with surfaces $2 \mathrm{~m}$ away from the ID PMT plane. The OD completely surrounds the ID and is instrumented with 1,885 20-cm diameter outward facing PMTs equipped with 60 $\mathrm{cm} \times 60 \mathrm{~cm}$ wavelength shifter plates to increase light collection efficiency. The main purpose of the OD is to tag incoming cosmic ray muons and exiting muons induced by atmospheric neutrinos. A detailed description of the Super-Kamiokande-I detector can be found elsewhere [14].

\section{DATA SET}

Data used for this analysis is taken during the period from May 1996 to July 2001. It corresponds to 1489 days of data taking and an exposure of $92 \mathrm{kt} \cdot$ year.

\section{NUCLEON DECAY EVENT AND BACKGROUND SIMULATION}

In order to search for nucleon decay in the SuperKamiokande detector, it is critical to understand the signal event signature and background characteristics. We simulate specific decay modes of nucleon decay as well as background events using a signal event generator and the standard Super-Kamiokande atmospheric Monte Carlo (MC). By comparing the characteristics of these signal and background simulated events in detail, we establish the optimum event selection criteria. When we limit the nucleon decay events to occur only in the Super-Kamiokande fiducial volume, the only significant background to nucleon decays originate from atmospheric neutrino interactions. Once the selection criteria are established, the detection efficiency is then estimated by analyzing the nucleon decay MC sample, and the expected background is estimated by analyzing the atmospheric neutrino MC sample.

\section{A. Nucleon decay event simulation}

Nucleon decay in water can occur from a free proton in the hydrogen nucleus or from a bound nucleon in the oxygen nucleus. It is relatively simple to simulate the free proton decay using the Super-Kamiokande MC. However, simulation of the bound nucleon decays requires special care, because of various nuclear effects experienced by the daughter particles from nucleon decay before they exit the nucleus.

Nucleons bound in oxygen have Fermi momentum and nuclear binding energy. In our simulation, we use the Fermi momentum distribution measured by an electron${ }^{12} \mathrm{C}$ scattering experiment 15 . For nucleon decays in an oxygen nucleus, the nucleon mass must be modified by nuclear binding energy. The modified nucleon mass $m_{N}^{\prime}$ is calculated by $m_{N}^{\prime}=m_{N}-E_{b i n d}$ where $m_{N}$ is the nucleon rest mass and $E_{\text {bind }}$ is the nuclear binding energy. Yamazaki and Akaishi [16] estimated the effective nucleon mass when the nucleon decays in ${ }^{16} \mathrm{O}$. Ten percent of decays are from a nucleus which has wave functions correlated with other nucleons in the nucleus. Figure 1 shows the invariant proton mass distribution in ${ }^{16} \mathrm{O}$ used in the $p \rightarrow \bar{\nu} K^{+}$MC simulation. The correlated decays produce the broad nucleon mass distribution below around $850 \mathrm{MeV} / c^{2}$.

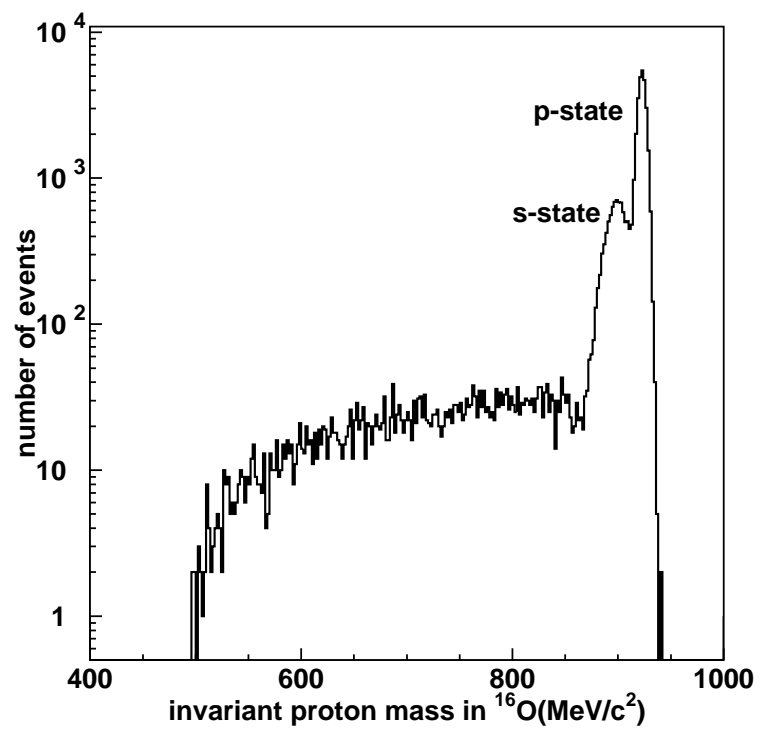

FIG. 1: The invariant proton mass distribution in ${ }^{16} \mathrm{O}$ in the $p \rightarrow \bar{\nu} K^{+}$MC simulation [16]. The distributions of s- and p-states are expressed by Gaussian function $G($ mean, $R M S)$. It is $G(938.3-39.0,10.2)) \mathrm{MeV} / c^{2}$ for s-state and $G(938.3-$ $15.5,3.82)) \mathrm{MeV} / c^{2}$ for p-state.

When a nucleon decays in the oxygen nucleus, the remaining nucleus can be in an excited state and it can emit prompt gamma-rays during its subsequent de-excitation process. This process was studied by Ejiri [17] and we use his results in this analysis. This prompt gamma-ray provides us with a powerful tool to tag $p \rightarrow \bar{\nu} K^{+}$events. 
The decay products of a nucleon bound in oxygen can interact hadronically with the remaining protons and neutrons in the residual ${ }^{15} \mathrm{~N}$ nucleus before exiting. The initial position of the nucleon is assumed to have the Woods-Saxon density distribution [18]:

$$
\rho(r)=\frac{\rho(0)}{1+\exp \left(\frac{r-a}{b}\right)},
$$

where $a\left(=1.07 \mathrm{~A}^{1 / 3}=2.69 \mathrm{fm}\right.$ for $\left.{ }^{16} \mathrm{O}\right)$ is the average of nuclear radius, $2 b(=0.82 \mathrm{fm})$ is the thickness of the nuclear surface, and $r$ is the distance from the center of the nucleus. Interactions relevant to this analysis are $K^{+} N$ and $K^{0} N$ elastic scatterings, and $K^{+} N$ and $K^{0} N$ inelastic scatterings via charge exchange. The $K^{+} p$ reactions can proceed elastically or inelastically. Inelastic interactions are due to the $K^{+} p \rightarrow K^{+} \Delta$. A partial wave analysis for the scattering amplitudes was performed by Hyslop et al. [19] by doing a global fit to many data samples. The maximum momentum for the kaon from the nucleon decays is about $600 \mathrm{MeV} / c$. Below $800 \mathrm{MeV} / c$, $K^{+} p$ scattering has an extremely small contribution from inelastic scattering. Therefore a $K^{+}$from a proton decay via $p \rightarrow \bar{\nu} K^{+}$effectively experiences only elastic scatterings with protons in the residual nucleus. Since $p \rightarrow \bar{\nu} K^{+}$ are identified through the detection of the daughter particles of the kaon decay at rest, $K^{+} p$ scattering does not affect the detection efficiency of $p \rightarrow \bar{\nu} K^{+}$. The charge exchange reaction $K^{+} n \rightarrow K^{0} p$ can reduce the efficiency for detecting $p \rightarrow \bar{\nu} K^{+}$events. It is important to estimate what fraction of $K^{+}$is lost due to this effect. The reaction was measured for low momentum kaons (250 to $600 \mathrm{MeV} / \mathrm{c}$ ) by Glasser et al [20] yielding cross sections ranging from $2.0 \pm 0.18 \mathrm{mb}$ at $K^{+}$momentum of $250 \mathrm{MeV} / c$ to $6.4 \pm 0.56 \mathrm{mb}$ at $K^{+}$momentum of 590 $\mathrm{MeV} / c$. To estimate the fraction of $K^{+}$lost due to this effect, a MC simulation is performed. $K^{+}$are started at random points in the nucleus according to the WoodsSaxon density distribution (Equation 11). If there is an interaction, Pauli blocking is taken into account by requiring the momentum of the recoil nucleon to be above the Fermi surface momentum $\left(p_{F}\right)$.

$$
p_{\text {recoil }}>p_{F}(r)=\hbar\left(\frac{3 \pi^{2}}{2} \rho(r)\right),
$$

where $\rho(r)$ is the same as defined in Equation 11 From this simulation, it is estimated that $1 \%$ of $K^{+}$from $p \rightarrow \bar{\nu} K^{+}$decays in total are lost because of this charge exchange reaction. From isospin symmetry, the $K^{0} N$ reactions have essentially the same magnitude as the $K^{+} N$ reactions.

We simulate propagation of the produced particles and Cherenkov light in water by custom code based on GEANT 21]. The propagation of charged pions in water is simulated by custom code based on [22] for less than $500 \mathrm{MeV} / c$ and by CALOR [24] for more than 500 $\mathrm{MeV} / c$.
In the $n \rightarrow \bar{\nu} K^{0}, p \rightarrow \mu^{+} K^{0}$ and $p \rightarrow e^{+} K^{0}$ searches, only decays to $K_{S}^{0}$ are studied because the lifetime of the $K_{L}^{0}$ is so long and many of them interact in water before decaying. The effect of $K^{0}$ regeneration is small in $K_{S}^{0}$ decay searches.

In total, 50,000 MC events are generated for each decay mode to find the signature of nucleon decays and to estimate detection efficiencies. Of these, 34,664, 34,561, 34,648 and 34,572 events are in the fiducial volume in the $p \rightarrow \bar{\nu} K^{+}, n \rightarrow \bar{\nu} K^{0}, p \rightarrow \mu^{+} K^{0}$ and $p \rightarrow e^{+} K^{0} \mathrm{MCs}$, respectively.

\section{B. Atmospheric neutrino background}

The most significant background to nucleon decay comes from the atmospheric neutrino interactions in the detector. Atmospheric neutrinos are produced in collisions of cosmic rays with air molecules in the atmosphere of the earth. Primary cosmic rays, mostly protons, interact hadronically with air molecules creating $\pi^{ \pm}, K^{ \pm}, K^{0}$, and other mesons. Neutrinos are then produced from the chain decay of these mesons. The production of atmospheric neutrinos has been calculated in great detail by many authors. For this analysis, we use the calculation by Honda et al [25].

Because the cross section for neutrinos to interact with matter is extremely small, they can travel unscattered through the earth and interact with a nucleon in the water of Super-Kamiokande via the weak interaction. A generic interaction is

$$
\nu_{l}+N \rightarrow l+N^{\prime}+X
$$

where $N$ and $N^{\prime}$ are the initial and final state nucleons, $l$ is the outgoing lepton associated with $\nu_{l}$, and $X$ can be other possible hadronic particles such as pions. Because some of these interactions result in topologies similar to those of nucleon decays, they present a challenging background to nucleon decay searches.

We use the same atmospheric neutrino MC simulation 26. that is used for Super-Kamiokande neutrino oscillation studies with slight modifications. The modifications made for this analysis are as following: for single pion production, the so-called axial vector mass which appears in the neutrino-nucleon differential cross section is set to be $1.2 \mathrm{GeV} / c^{2}$ to estimate conservatively. For deep inelastic scattering, we use a model motivated by Bodek and Yang [27].

In order to estimate the atmospheric neutrino background events for the nucleon decays involving kaons, the production of kaons through the baryon resonances is included based on the model of Rein and Sehgal [28]. The cross section for kaon production is one or more than one order of magnitude smaller than that of single pion production. In addition, many charged kaons produced in neutrino interactions emit Cherenkov light while kaons from proton decay do not, and $K$ production is accompanied by a $\Lambda$ baryon which decays into either $p \pi^{-}$or $n \pi^{0}$ 
in most cases. Therefore these background events can be distinguished from nucleon decay events, and their contributions to the background can be safely ignored.

In order to estimate the backgrounds from the atmospheric neutrinos to nucleon decays at a high precision, we use a total of 100 year equivalent sample of atmospheric neutrino MC simulation events for this analysis.

\section{EVENT SELECTION AND RECONSTRUCTION}

We apply the first stage reduction for the data to remove major background. Then we reconstruct physical quantities for data and also signal and background MC events. Data reduction and event reconstruction is the same as for the neutrino oscillation analysis [26].

\section{A. Event Selection}

The trigger threshold for events used in this analysis is set to 29 PMT hits corresponding to an electron equivalent energy of $5.7 \mathrm{MeV}$. The trigger rate at this threshold is about $10 \mathrm{~Hz}$. The majority of the collected data are the events originating from cosmic muons and radioactivities. An event selection process is applied to remove these events. Almost all cosmic muons are rejected requiring no significant OD activities. After applying event reconstruction, events are rejected when their electron equivalence energy is less than $30 \mathrm{MeV}$ corresponding to $197 \mathrm{MeV} / \mathrm{c}$ for muons or when the event vertex is located outside of the fiducial volume. The remaining event rate after this first stage reduction is about eight events/day. Almost all of these events originate from atmospheric neutrino interaction.

\section{B. Event Reconstruction}

Physical quantities are reconstructed for the events remaining from the event selection process. In the reconstruction, certain quantities are determined such as the event vertex, the number of visible Cherenkov rings, the direction of each ring, particle identification, momentum and number of Michel electrons. The vertex resolution is $34 \mathrm{~cm}$ for electrons and $25 \mathrm{~cm}$ for muons in single ring events. In the $p \rightarrow \bar{\nu} K^{+}, K^{+} \rightarrow \mu^{+} \nu_{\mu}$ and $K^{+} \rightarrow \pi^{+} \pi^{0}$ $\mathrm{MC}$, the vertex resolution is 47 and $37 \mathrm{~cm}$, respectively. In the $n \rightarrow \bar{\nu} K^{0}, p \rightarrow \mu^{+} K^{0}$ and $p \rightarrow e^{+} K^{0} \mathrm{MC}$, better resolutions are obtained. Each ring is identified as $e$-like $\left(\mathrm{e}^{ \pm}, \gamma\right)$ or $\mu$-like $\left(\mu^{ \pm}, \pi^{ \pm}\right.$,proton $)$based on a likelihood analysis of Cherenkov ring pattern and Cherenkov angle. In a single ring electron or muon event, the misidentification probability is $0.5 \%$. Momentum is determined by the sum of photo electrons (PEs) after correcting for light attenuation in water, PMT angular acceptance, PMT coverage, and the assigned electron, muon, or pion particle assumption. The momentum of a pion is determined by Cherenkov angle as well as the sum of PEs. The momentum resolution for a $236 \mathrm{MeV} / \mathrm{c}$ muon from $p \rightarrow \bar{\nu} K^{+}, K^{+} \rightarrow \mu^{+} \nu_{\mu}$ is $3 \%$ and mass resolution for $\pi^{0}$ from $p \rightarrow \bar{\nu} K^{+}, K^{+} \rightarrow \pi^{+} \pi^{0}$ is 9\%. Figure 2 shows the reconstructed $\pi^{0}$ invariant mass distribution in the $p \rightarrow \bar{\nu} K^{+}, K^{+} \rightarrow \pi^{+} \pi^{0}$ MC. The detection efficiency of Michel electrons is estimated to be $80 \%$ for $\mu^{+}$ and $63 \%$ for $\mu^{-}$.

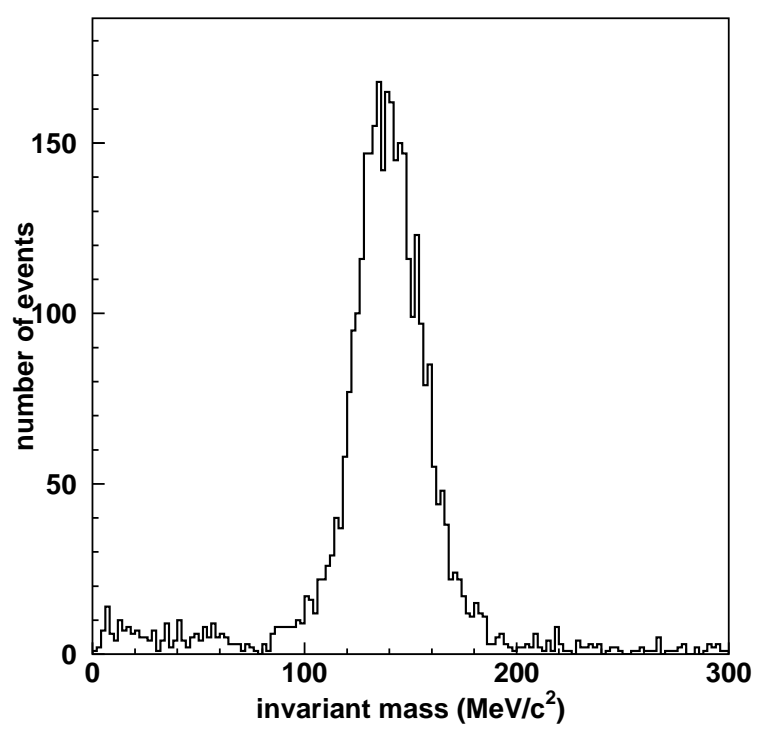

FIG. 2: The reconstructed $\pi^{0}$ invariant mass distribution in the $p \rightarrow \bar{\nu} K^{+}, K^{+} \rightarrow \pi^{+} \pi^{0} \mathrm{MC}$ after applying selection criteria (B1,B2) (See Section VIA).

\section{Calibration}

An important aspect of nucleon decay analyses is the choice of selection criteria. These criteria are dependent upon the energies of the particles produced in the various interactions. For this reason, the energy deposition and detector response of particles traversing the detector must be accurately modeled in the MC simulation. Energy scale calibrations are performed by comparing the data and MC sample of Michel electrons from stopping cosmic ray muons, $\pi^{0}$ s produced in atmospheric neutrino interactions and stopping cosmic ray muons. The Cherenkov angle and the range are used to obtain muon momentum for stopping muons. These samples provide energy calibration varying from several tens $\mathrm{MeV}$ (Michel electrons) to several $\mathrm{GeV}$ (stopping cosmic ray muons), entirely covering the energy range relevant to nucleon decay searches. Based on these samples, the energy scale is reproduced in the MC within $2.5 \%$. 


\section{ANALYSIS}

During 1,489 days of livetime corresponding to 92 kton·year of exposure, 12,179 events are recorded after the first stage reduction process. Of these, 8,207 are identified as single ring.

It has been well known for some time that there is a deficit of events induced by atmospheric muon neutrino oscillations [10]. In order to estimate accurately the actual number of background events, the atmospheric neutrino flux is normalized based on the observed deficit of $\mu$-like events due to well established neutrino oscillation phenomena and the observed number of $e$-like events. The normalizing factor is determined as multiplying 0.67 for charged current $\nu_{\mu}$ events and 1.07 for charged current $\nu_{e}$ and all neutral current events. Finally we normalize the 100 year MC sample (2,246 kton-years) to the 92 kton·year sample of Super-Kamiokande exposure. Based on these simulated signal and background events, we determine selection criteria for each nucleon decay mode, which is discussed in the following sections. All detection efficiencies given below include all relevant branching ratios.

\section{A. Search for $p \rightarrow \bar{\nu} K^{+}$}

This is the primary mode of proton decay in SUSY GUT models. A search for $p \rightarrow \bar{\nu} K^{+}$was described in our previous paper 29] based on the $45 \mathrm{kton} \cdot$ year data. In that paper, we described three methods to search for this mode using the two dominant decay modes of the kaon, $K^{+} \rightarrow \pi^{+} \pi^{0}$ (Method 1) and $K^{+} \rightarrow \mu^{+} \nu_{\mu}$. For $K^{+} \rightarrow \mu^{+} \nu_{\mu}$, we looked for both the prompt gammaray from the de-excitation of the residual excited ${ }^{15} \mathrm{~N}$ nucleus (Method 2: prompt gamma-ray search) and for an excess in the momentum distribution of $\mu$-like events at $p_{\mu}=236 \mathrm{MeV} / c$ (Method 3: mono-energetic muon search). In this paper, we present proton lifetime limits derived using these methods including improved analyses which increase the detection efficiency and reduce the background.

\section{Method 1: $K^{+} \rightarrow \pi^{+} \pi^{0}$ search}

If a proton decays to $\bar{\nu} \mathrm{K}^{+}$, the $\mathrm{K}^{+}$has low enough momentum that the majority stop before decaying. Therefore, when the $\mathrm{K}^{+}$decays to a $\pi^{+}$and a $\pi^{0}$, these two particles go back-to-back and the $\pi^{0}$ momentum is expected to be mono-energetic at $205 \mathrm{MeV} / c$. To detect this type of events, the following criteria are required: (A1) two $e$-like rings, (A2) one Michel electron, (A3) $175 \mathrm{MeV} / c<p_{\pi^{0}}<250 \mathrm{MeV} / c$, (A4) 85 $\mathrm{MeV} / c^{2}<m_{\pi^{0}}<185 \mathrm{MeV} / c^{2}$, (A5) $40 \mathrm{PE}<Q_{\pi^{+}}<100$ PE, (A6) $Q_{\text {res }}<70$ PE. The reconstructed total momentum and invariant mass consistent with the $\pi^{0}$ from the two $e$-like rings are defined as $p_{\pi^{0}}$ and $m_{\pi^{0}}$, respectively.
The $\pi^{+}$momentum is so close to the Cherenkov threshold that the Cherenkov ring is not detected in most cases. However, since many backgrounds survive criteria (A1A4), we use the Cherenkov light produced by the $\pi^{+}$in addition. $Q_{\pi^{+}}$is sum of PEs corrected or light attenuation and PMT acceptance, which is observed in the PMTs within $40^{\circ}$ half opening angle opposite to the $\pi^{0}$ direction. $Q_{\text {res }}$ is sum of PEs in the remaining PMTs after rejecting the area within the $90^{\circ}$ half opening angle toward the two gamma-ray directions and the $Q_{\pi^{+}}$ searched area. We use the criterion (A6) for background rejection. The total detection efficiency for this method is estimated to be $6.0 \%$ and the background is estimated to be 0.6 events. Figure 3 shows the distribution of the $Q_{\pi^{+}}$versus $p_{\pi^{0}}$ for data, the atmospheric neutrino MC and $p \rightarrow \bar{\nu} K^{+}$MC. The main sources of background are events by single pion production and deep inelastic scattering. The incoming neutrino energy of the background is typically between $0.6 \mathrm{GeV}$ and $2 \mathrm{GeV}$. In the data, no events pass the selection criteria (A1-A6).

\section{Method 2: $K^{+} \rightarrow \mu^{+} \nu_{\mu}$, prompt gamma-ray search}

When a proton decays in one of the inner shells of the ${ }^{16} \mathrm{O}$ nucleus, the residual ${ }^{15} \mathrm{~N}$ nucleus is left in an excited state. This state quickly de-excites with a certain probability of emitting gamma-rays. The most probable residual state is a $p_{3 / 2} \quad 6.3 \mathrm{MeV}$ state which leads to an emission of single $6.3 \mathrm{MeV}$ gamma-ray [17]. Since the $\mathrm{K}^{+}$is below the Cherenkov threshold and the $\mathrm{K}^{+}$ lifetime is $12.4 \mathrm{~ns}$, we can separate the de-excitation gamma-ray from the $\mu^{+}$signal. The $\mu^{+}$momentum is mono-energetic $(236 \mathrm{MeV} / c)$, because the $\mathrm{K}^{+}$decays at rest. By requiring a prompt gamma-ray signal as well as the mono-energetic muon and an electron from the muon decay, most backgrounds are eliminated.

In order to search for the prompt gamma-ray, three quantities must be defined. The first is $t_{\mu}$ which is a reference time associated with the detection of the muon. The second is $t_{0}$ which is the time to begin a backward search in time of flight (TOF) subtracted timing distribution for the earlier hits from the prompt gamma-ray. Finally, $t_{\gamma}$ is the time associated with the detection of the gamma-ray. The reference point $t_{\mu}$ corresponding to the muon is found by searching for the point in time when $\Delta N_{\text {hit }} / \Delta t$ is maximum where $N_{\text {hit }}$ is number of PMT hits. The starting time $t_{0}$ is defined as the first point less than $t_{\mu}$ where $d N_{h i t} / d t=0$. In the $t_{\gamma}$ search, PMTs which are within a cone with a $50^{\circ}$ half opening angle with respect to the muon are removed. Removing these tubes enables the search for the prompt gamma-ray to start at a closer time to $t_{\mu}$. A 12 ns timing window is slid backward starting with its trailing edge at $t_{0}$. The values $t_{\gamma}$ and $N_{h i t \gamma}$ are determined by maximizing the number of hits in the $12 \mathrm{~ns}$ sliding window. $N_{\text {hit }}$ is the maximum number of hits at $t_{\gamma}$ in the 12 ns sliding window. 


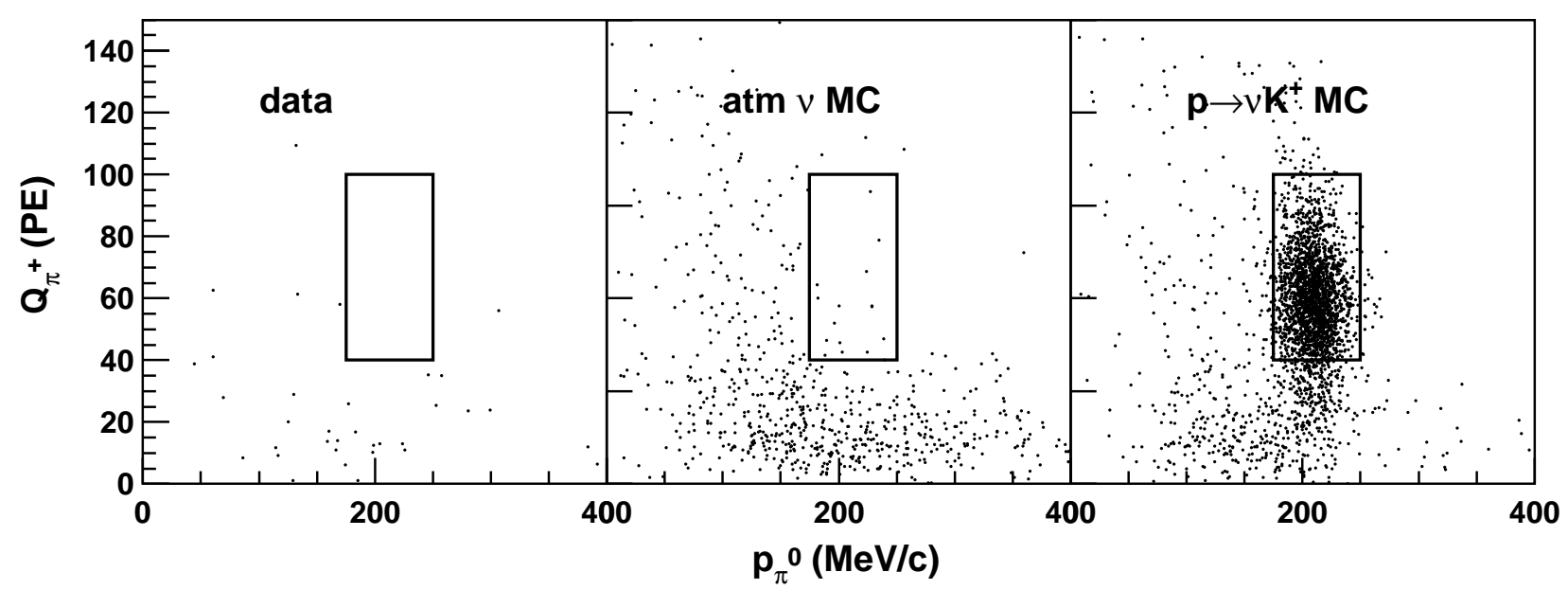

FIG. 3: The distributions of the $Q_{\pi^{+}}$versus $p_{\pi^{0}}$ for events that satisfy criteria (A1,A2,A4,A6). Each figure shows: (left) data, (middle) atmospheric neutrino $\mathrm{MC}$ and (right) $p \rightarrow \bar{\nu} K^{+} \mathrm{MC}$. The box shows criteria (A3,A5).

Using these quantities, the following selection criteria are applied: (B1) one $\mu$-like ring, (B2) one Michel electron, (B3) $215 \mathrm{MeV} / c<p_{\mu}<260 \mathrm{MeV} / c$, (B4) proton rejection, (B5) $t_{\mu}-t_{\gamma}<100 \mathrm{ns,} \mathrm{(B6)} 7<N_{\text {hit } \gamma}<$ 60. Criterion (B4) is applied for rejecting backgrounds caused by poor vertex reconstruction. Most of this type of backgrounds is recoil protons produced by neutral current interactions. Since the particle type is assumed as a muon in the vertex fit, the reconstructed vertex position of a proton is not accurate. Therefore a fake peak, which mimics gamma-rays, is sometimes produced in the TOF subtracted timing distribution. In order to reject these events, two cuts, $g \geq 0.6$ and $d_{\mu e}<200 \mathrm{~cm}$, are applied, where $g$ is a goodness of the TOF subtracted timing distribution and $d_{\mu e}$ is the distance between the Michel positron vertex and the muon stopping point. In proton decay events, $d_{\mu e}$ should be close to zero.

Passing the $p \rightarrow \bar{\nu} K^{+}$and 100 year atmospheric neutrino MC events through criteria (B1-B6), the detection efficiency and background are estimated to be $8.6 \%$ and 0.7 events, respectively. The efficiency includes all branching ratios. Figure 4 shows $N_{\text {hit }}$ distribution for the 100 year sample of atmospheric neutrino MC normalized by livetime and neutrino oscillation, $p \rightarrow \bar{\nu} K^{+} \mathrm{MC}$, and $92 \mathrm{kton} \cdot \mathrm{yr}$ sample of data. No events survive these selection criteria.

\section{Method 3: $K^{+} \rightarrow \mu^{+} \nu_{\mu}$, mono-energetic muon search}

We search for a $236 \mathrm{MeV} / c$ mono-energetic muon using events which are not selected in Method 2. To tag the muon, criteria $(\mathrm{B} 1, \mathrm{~B} 2)$ are applied, which are defined as in Method 2. Moreover, to obtain an independent event sample from Method 2, we apply criterion (B7) $N_{\text {hit } \gamma} \leq 7$. Figure 5 shows the muon momentum distribution of data compared with best fitted atmospheric neutrino MC. No significant excess is observed in the sig-

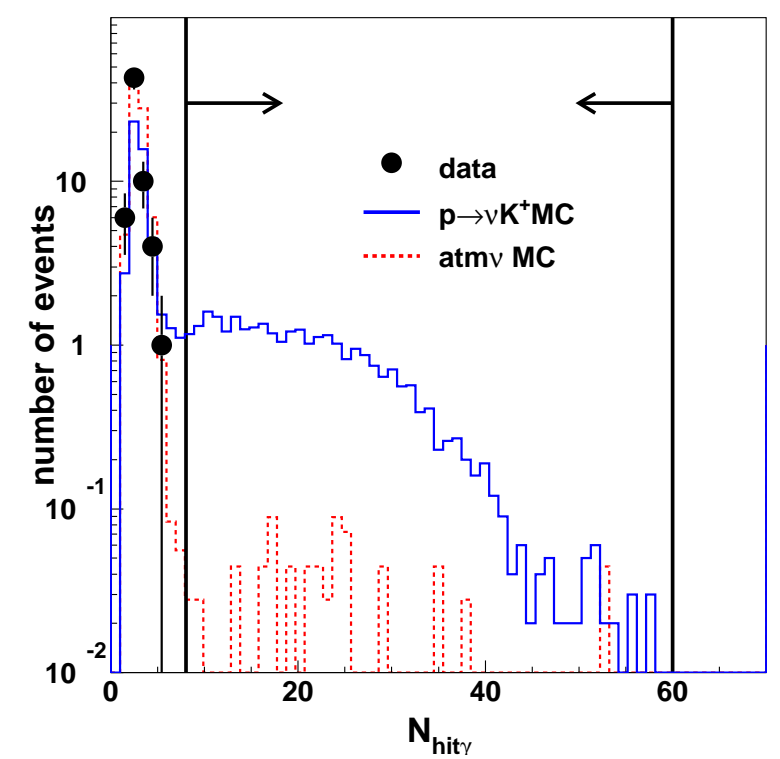

FIG. 4: The $N_{\text {hit } \gamma}$ distributions for events that satisfy criteria (B1-B5). The full circles show data and the dashed line shows atmospheric neutrino MC normalized by livetime and neutrino oscillation. The solid line shows $p \rightarrow \bar{\nu} K^{+} \mathrm{MC}$. The arrows show criterion (B6), where proton decay candidates with a prompt gamma-ray are expected.

nal region (B3). In this region, there are 181 events with a best fitted background of 200 events.

\section{B. Search for $n \rightarrow \bar{\nu} K^{0}$}

Many SUSY models also predict the nucleon decay mode, $n \rightarrow \bar{\nu} K^{0}$. We search for $n \rightarrow \bar{\nu} K^{0}$ using the $K_{S}^{0} \rightarrow \pi^{0} \pi^{0}$ and $K_{S}^{0} \rightarrow \pi^{+} \pi^{-}$decay chain.

For the $K_{S}^{0} \rightarrow \pi^{0} \pi^{0}$ search, the following criteria are applied: (C1) three or four $e$-like rings, (C2) zero Michel 


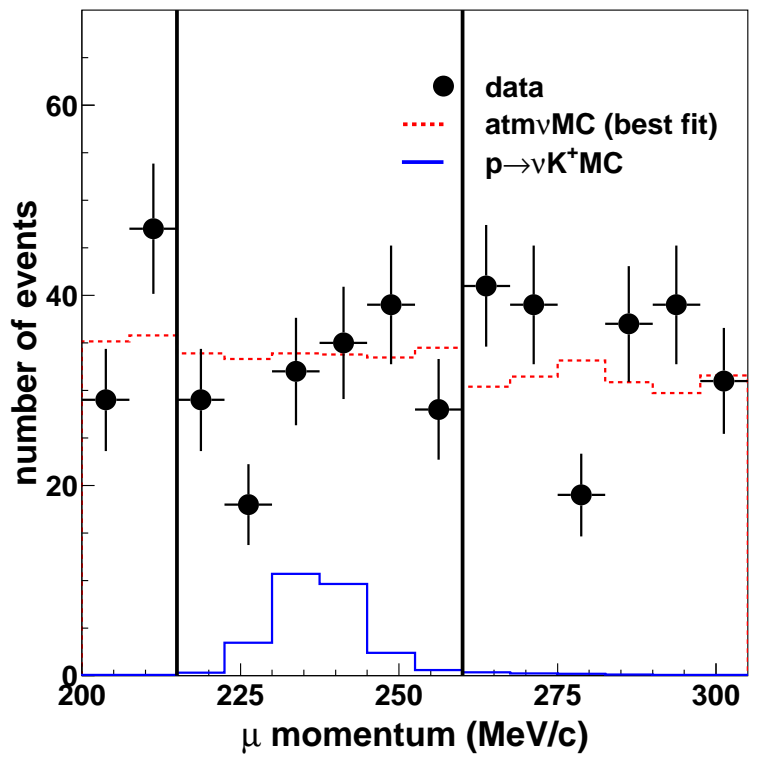

FIG. 5: The comparison between data and fitting results of muon momentum distribution for events that satisfy criteria (B1,B2,B7). The full circles show data with statistical errors. The solid line shows $p \rightarrow \bar{\nu} K^{+}$MC. The dashed line shows the best fitted atmospheric neutrino $\mathrm{MC}$ with free normalization.

electrons, (C3) $200 \mathrm{MeV} / c<p_{K^{0}}<500 \mathrm{MeV} / c$, (C4) $400 \mathrm{MeV} / c^{2}<m_{K^{0}}<600 \mathrm{MeV} / c^{2}$, where $p_{K^{0}}$ and $m_{K^{0}}$ are total momentum and invariant mass, assuming the decay sequence of $K^{0}$. Because it is difficult to identify four showering Cherenkov rings, many background events remain. About half of the background events come from deep inelastic scattering. Figure [ 6 shows $p_{K^{0}}$ versus $m_{K^{0}}$ distributions after applying criteria $(\mathrm{C} 1, \mathrm{C} 2)$. The criteria select 14 events in the data with an expected background of 19 and a selection efficiency of $6.9 \%$.

For the $K_{S}^{0} \rightarrow \pi^{+} \pi^{-}$search, the following criteria are applied: (D1) two $\mu$-like rings, (D2) zero or one Michel electron, (D3) $200 \mathrm{MeV} / c<p_{K^{0}}<500 \mathrm{MeV} / c$, (D4) 450 $\mathrm{MeV} / c^{2}<m_{K^{0}}<550 \mathrm{MeV} / c^{2}$. Since a large fraction of $\pi^{+}$or $\pi^{-}$momenta are below the Cherenkov threshold, the efficiency of finding both rings is low. Figure $\mathbf{7}$ shows $p_{K^{0}}$ versus $m_{K^{0}}$ distributions after applying criteria $(\mathrm{D} 1, \mathrm{D} 2)$. Twenty events are observed in the data with the detection efficiency of $5.5 \%$, while 11.2 background events are expected. Most of the background events are produced by charged current single pion production.

\section{Search for $p \rightarrow \mu^{+} K^{0}$}

In a SUSY $S O(10)$ model [11] with neutrino mass, $p \rightarrow \mu^{+} K^{0}$ is an important decay mode. We search for $p \rightarrow \mu^{+} K^{0}$ using $K_{S}^{0} \rightarrow \pi^{0} \pi^{0}$ and $K_{S}^{0} \rightarrow \pi^{+} \pi^{-}$decay chain. In this mode, the total invariant proton mass and momentum can be reconstructed and the backgrounds can be significantly reduced.

For the $K_{S}^{0} \rightarrow \pi^{0} \pi^{0}$ search, the following criteria are applied: (E1) $2-4 e$-like rings and one $\mu$-like ring, (E2) zero or one Michel electron, (E3) $400 \mathrm{MeV} / c^{2}<m_{K^{0}}<$ $600 \mathrm{MeV} / c^{2}$, (E4) $150 \mathrm{MeV} / c<p_{\mu}<400 \mathrm{MeV} / c$, (E5) $p_{p}<300 \mathrm{MeV} / c$, (E6) $750 \mathrm{MeV} / c^{2}<m_{p}<1000$ $\mathrm{MeV} / c^{2}$, where $m_{p}$ and $p_{p}$ are the invariant mass and total momentum assuming the decay sequence of proton, respectively. The momentum of the $\mu^{+}, p_{\mu}$, is determined using the $\mu$-like ring. Figure 8 shows $p_{p}$ versus $m_{p}$ distributions after applying criteria (E1-E4). No events survive in the data with the estimated $5.4 \%$ detection efficiency and 0.4 events of the expected background.

For the $K_{S}^{0} \rightarrow \pi^{+} \pi^{-}$search, two different methods are applied, because ring finding efficiency of the two pions are low. The first method (Method 1) uses the following criteria: (F1) two $\mu$-like rings, (F2) two Michel electrons, (F3) $250 \mathrm{MeV} / c<p_{\mu}<400 \mathrm{MeV} / c$, (F4) $p_{p}<300$ $\mathrm{MeV} / c$. We assume the more energetic ring as muon and the other as charged pion. Figure 9 shows $p_{p}$ versus $p_{\mu}$ distributions after applying criteria (F1,F2). The detection efficiency and the expected background are $7.0 \%$ and 3.2 events, respectively; 3 events are observed in the data. The second method (Method 2) requires: (G1) three rings, (G2) one or two Michel electrons, (G3) 450 $\mathrm{MeV} / c^{2}<m_{K^{0}}<550 \mathrm{MeV} / c^{2},(\mathrm{G} 4) p_{p}<300 \mathrm{MeV} / c$, (G5) $750 \mathrm{MeV} / c^{2}<m_{p}<1000 \mathrm{MeV} / c^{2}$. Figure 10 shows the $p_{p}$ versus $m_{p}$ distributions after applying criteria (G1-G3). The detection efficiency and the expected background are estimated to be $2.8 \%$ and 0.3 events, respectively. Most of the background events come from charged current $\nu_{\mu}$ single pion production. No surviving events are observed in the data.

\section{Search for $p \rightarrow e^{+} K^{0}$}

One of the supersymmetric theories, based on flavor group $\left(S_{3}\right)^{3}$ predicts that $p \rightarrow e^{+} K^{0}$ occurs at a comparable rate with $p \rightarrow \bar{\nu} K^{+}$and $n \rightarrow \bar{\nu} K^{0}$ [30]. For the search for $p \rightarrow e^{+} K^{0}$ mode, three methods are applied in the same way as $p \rightarrow \mu^{+} K^{0}$ search. For the $K_{S}^{0} \rightarrow \pi^{0} \pi^{0}$ search, the selection criteria are: (H1) 3-5 e-like rings, (H2) zero Michel electrons, (H3) $p_{p}<300 \mathrm{MeV} / c$, (H4) $750 \mathrm{MeV} / c^{2}<m_{p}<1000 \mathrm{MeV} / c^{2}$. We assume the most energetic ring as electron and others as gamma-rays from the $\pi^{0}$ decays. Figure 11 shows $p_{p}$ versus $m_{p}$ distributions after applying criteria $(\mathrm{H} 1, \mathrm{H} 2)$. The detection efficiency and the expected background are $9.2 \%$ and 1.1 events, respectively. One candidate event is observed in the data.

For the $K_{S}^{0} \rightarrow \pi^{+} \pi^{-}$search, two different methods are applied. The first method (Method 1) uses the following criteria: (I1) one $\mu$-like ring and one $e$-like ring, (I2) one Michel electron, (I3) $250 \mathrm{MeV} / c<p_{e}<400 \mathrm{MeV} / c$, (I4) $p_{p}<300 \mathrm{MeV} / c$, where $p_{e}$ is the electron momentum determined using the $e$-like ring. Figure 12 shows $p_{p}$ versus $p_{e}$ distributions after applying criteria (I1,I2). The detection efficiency and the expected background are 7.9\% and 3.6 events, respectively. Most of the background 


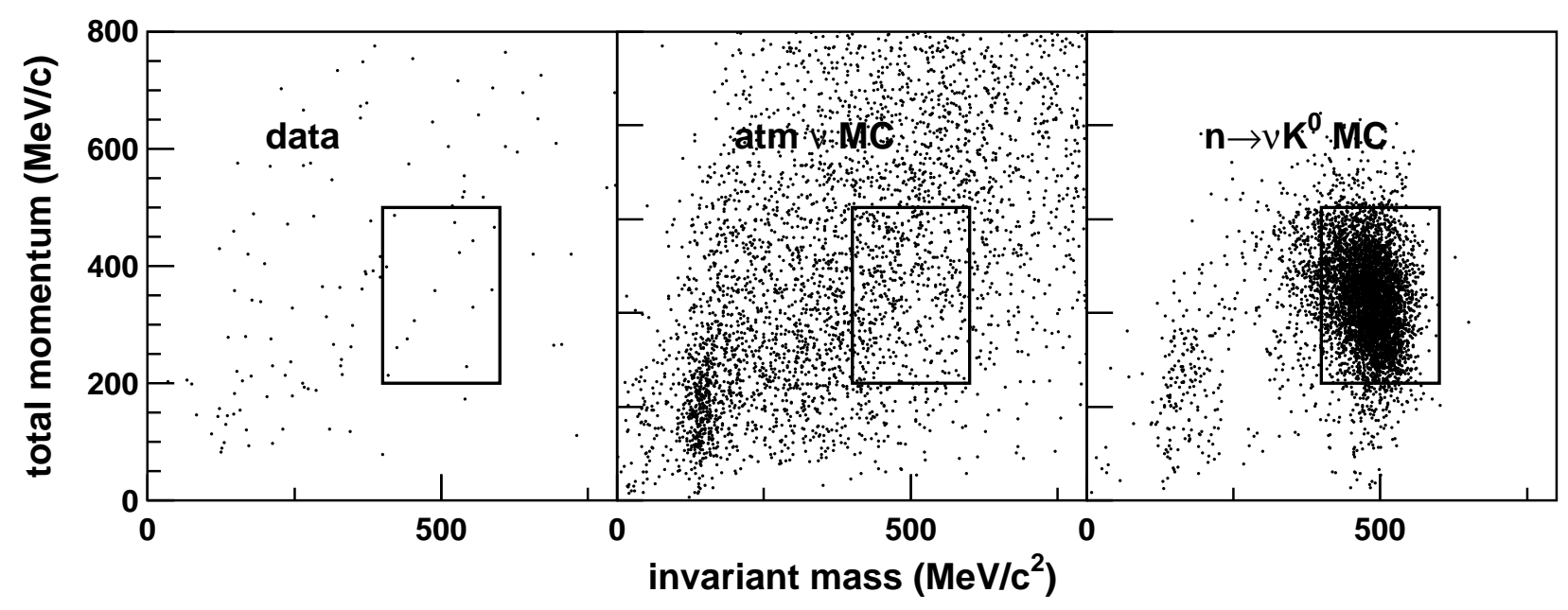

FIG. 6: The distributions of the total momentum versus invariant mass for events that satisfy criteria (C1,C2). Each figure shows: (left) data, (middle) atmospheric neutrino MC and (right) $n \rightarrow \bar{\nu} K^{0}$ MC. The box shows criteria (C3,C4).

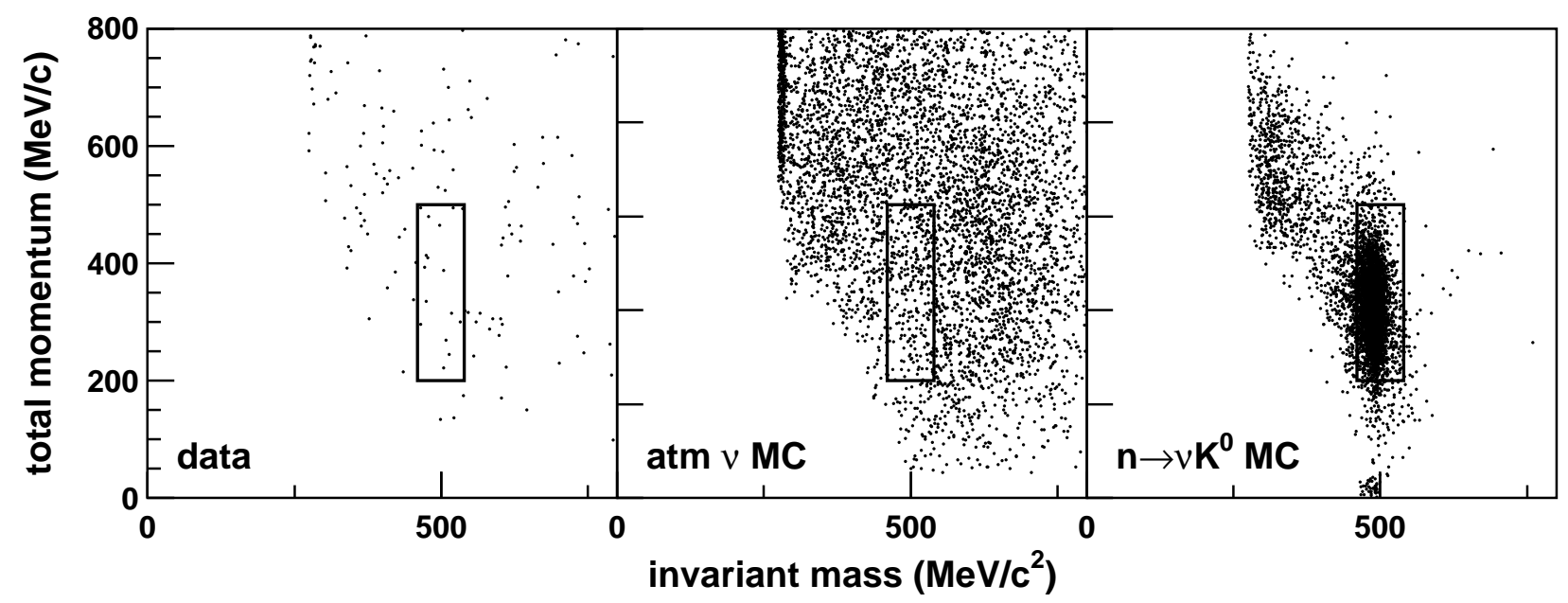

FIG. 7: The distributions of the total momentum versus invariant mass for events that satisfy criteria (D1,D2). Each figure shows: (left) data, (middle) atmospheric neutrino $\mathrm{MC}$ and (right) $n \rightarrow \bar{\nu} K^{0} \mathrm{MC}$. The box shows criteria (D3,D4). The cluster in the atmospheric neutrino MC figure comes from mis-reconstruction.

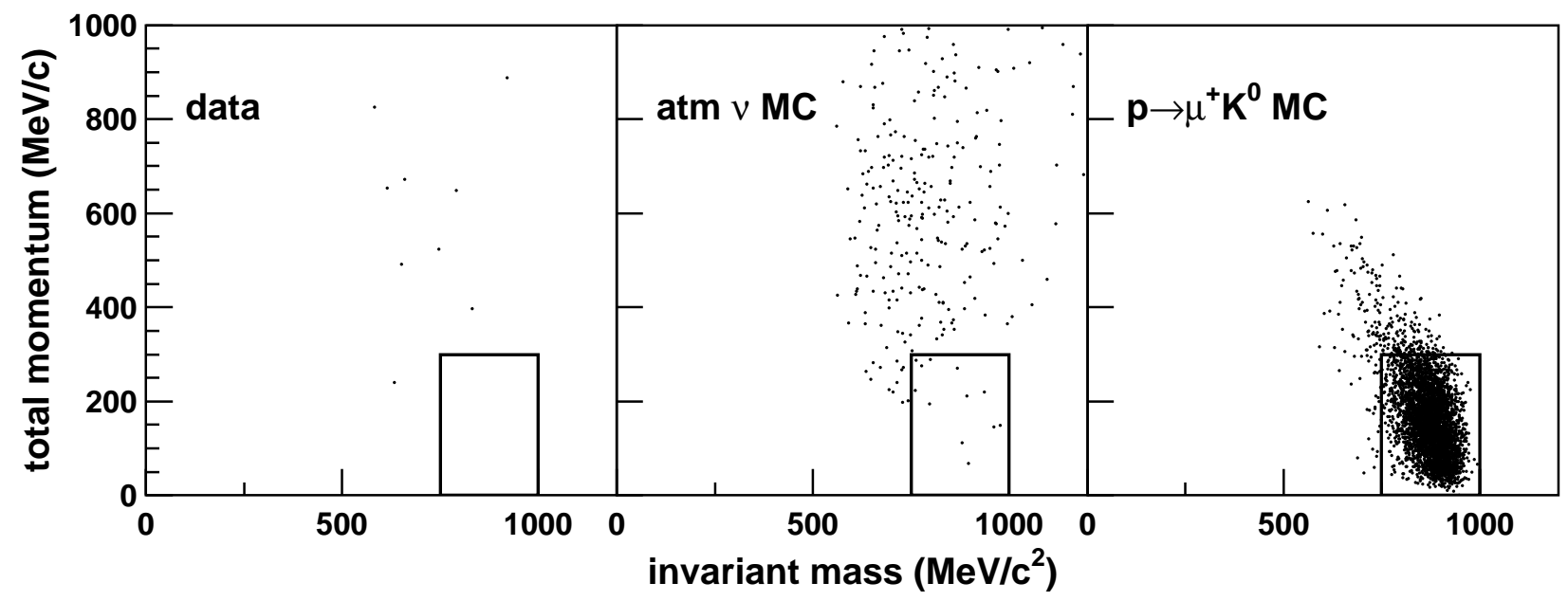

FIG. 8: The distributions of the total momentum versus invariant mass for events that satisfy criteria (E1-E4). Each figure shows: (left) data, (middle) atmospheric neutrino MC and (right) $p \rightarrow \mu^{+} K^{0} \mathrm{MC}$. The box shows criteria (E5,E6). 


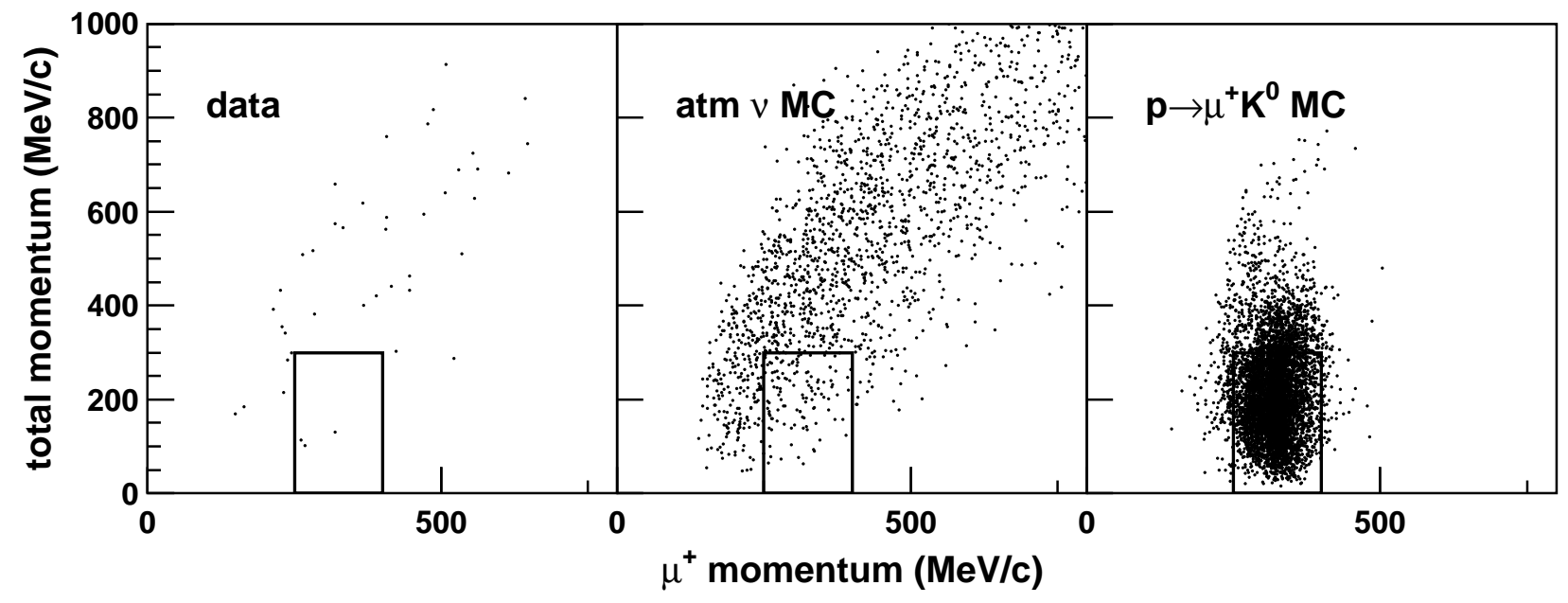

FIG. 9: The distributions of the total momentum versus $\mu^{+}$momentum for events that satisfy criteria (F1,F2). Each figure shows: (left) data, (middle) atmospheric neutrino MC and (right) $p \rightarrow \mu^{+} K^{0} \mathrm{MC}$. The box shows criteria (F3,F4).

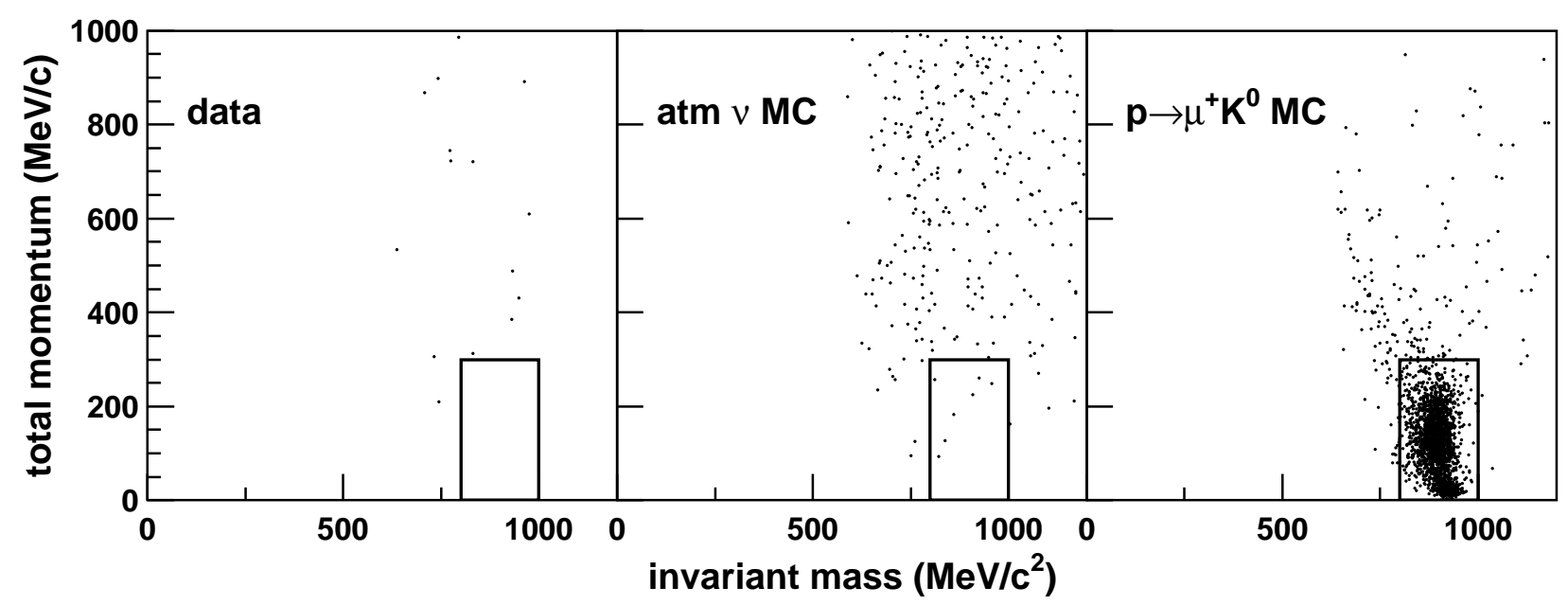

FIG. 10: The distributions of the total momentum versus invariant mass for events that satisfy criteria (G1-G3). Each figure shows: (left) data, (middle) atmospheric neutrino MC and (right) $p \rightarrow \mu^{+} K^{0} \mathrm{MC}$. The box shows criteria (G4,G5).

events are produced by charged current $\nu_{e}$ single pion events. Five events are observed in the data. The second method (Method 2) uses the criteria: (J1) three rings (at least one $e$-like), (J2) zero or one Michel electron, (J3) $450 \mathrm{MeV} / c^{2}<m_{K^{0}}<550 \mathrm{MeV} / c^{2}$, (J4) $p_{p}<300$ $\mathrm{MeV} / c$, (J5) $750 \mathrm{MeV} / c^{2}<m_{p}<1000 \mathrm{MeV} / c^{2}$. Figure 13 shows $p_{p}$ versus $m_{p}$ distributions after applying criteria (J1-J3). The detection efficiency is estimated to be $1.3 \%$ with 0.04 expected backgrounds. No surviving events are observed in the data.

\section{E. Systematic uncertainties}

In the detection efficiency, we consider the following common systematic uncertainties in every search: imperfect knowledge of light scattering in water, energy scale, and particle identification. The uncertainty of the rate of light scattering in water is estimated to be $20 \%$, which mainly effects on the ring finding efficiency. The energy scale uncertainty is estimated to be $2.5 \%$ by the calibration described in Section $\mathrm{VC}$. For the modes which have a charged pion from kaon decay, we consider the imperfect knowledge of a charged pion-nucleon cross section in water which is estimated to be $10 \%$ by comparing with our MC and experimental data 23. For the $p \rightarrow \mu^{+} K^{0}$ and $p \rightarrow e^{+} K^{0}$ search, we consider the uncertainty of the Fermi momentum which is estimated to be about $5 \%$ from model differences [15]. In addition, we consider the uncertainty of de-excitation gamma-ray emission probabilities in the $p \rightarrow \bar{\nu} K^{+}$, prompt gamma-ray search. It is estimated to be $15 \%$ for $6.3 \mathrm{MeV}$ gamma-ray and $30 \%$ for other gamma-rays [17]. From these sources of systematic uncertainty, the total contribution to the uncertainty of the detection efficiency in the $p \rightarrow \bar{\nu} K^{+}$, prompt gamma-ray search is estimated to be $20 \%$. The main 


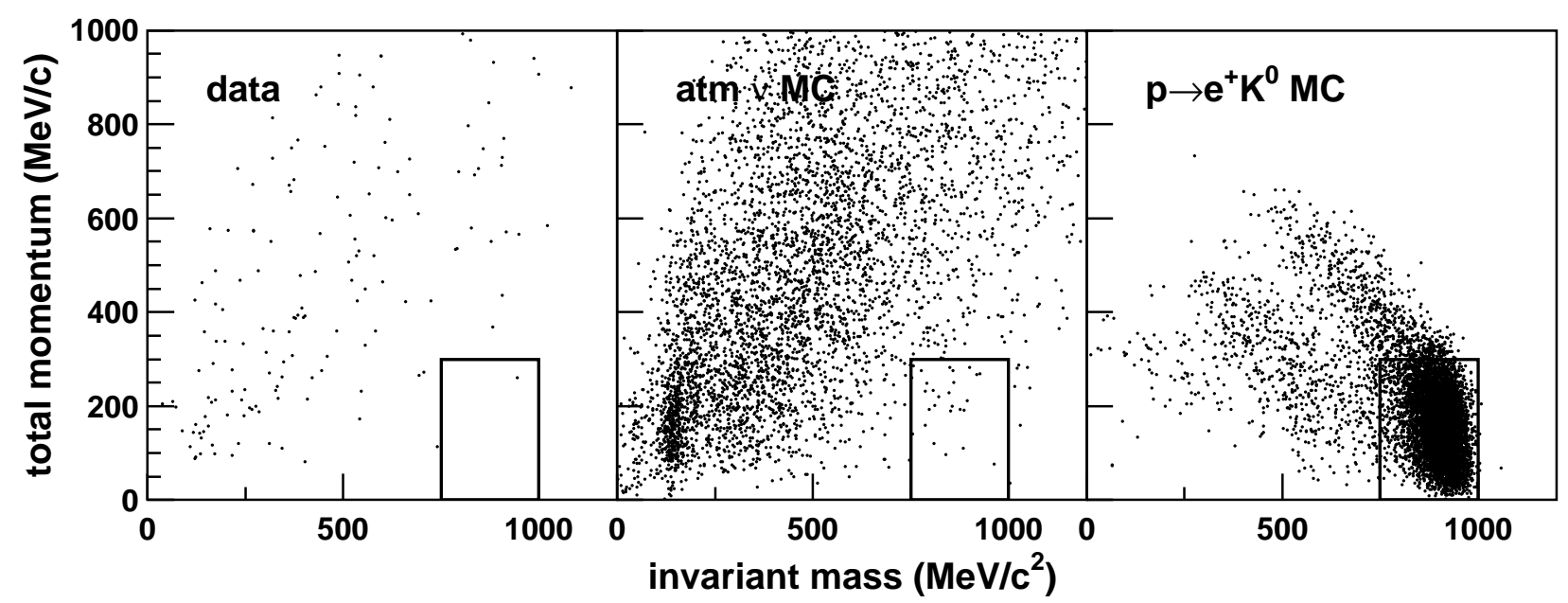

FIG. 11: The distributions of the total momentum versus invariant mass for events that satisfy criteria (H1,H2). Each figure shows: (left) data, (middle) atmospheric neutrino MC and (right) $p \rightarrow e^{+} K^{0} \mathrm{MC}$. The box shows criteria (H3,H4).

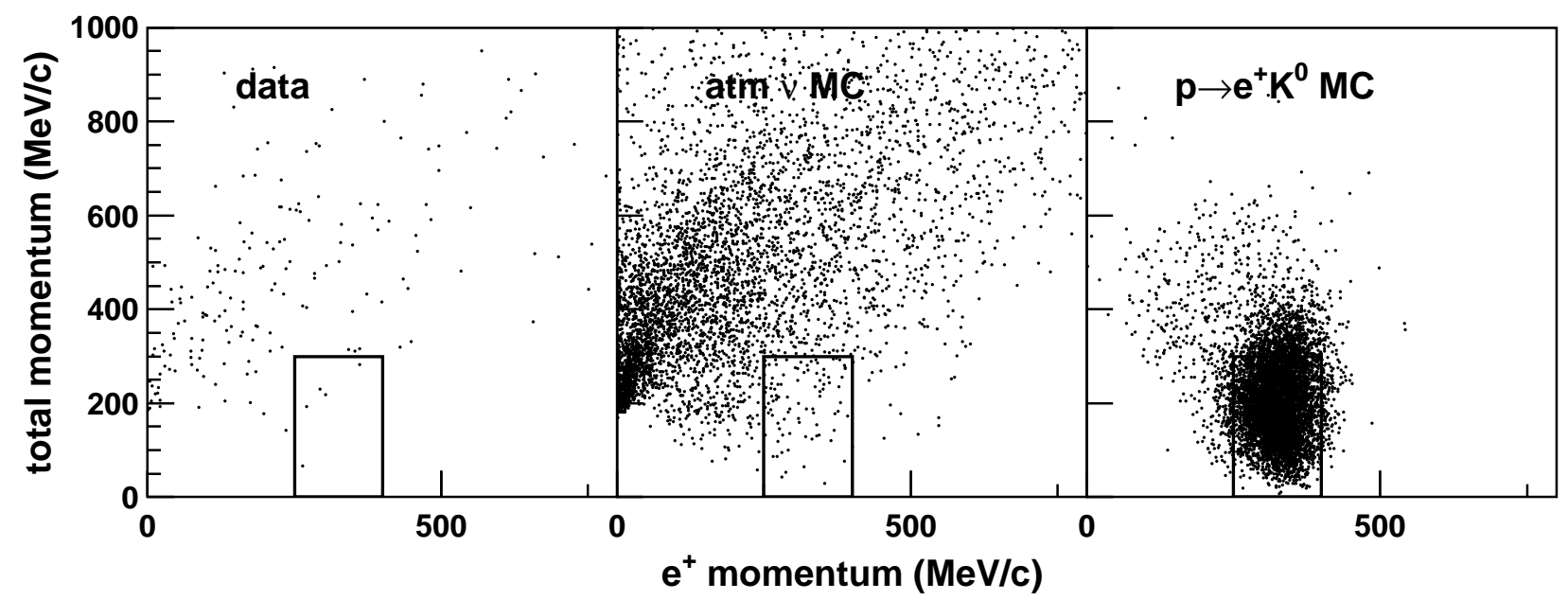

FIG. 12: The distributions of the total momentum versus $e^{+}$momentum for events that satisfy criteria (I1,I2). Each figure shows: (left) data, (middle) atmospheric neutrino MC and (right) $p \rightarrow e^{+} K^{0} \mathrm{MC}$. The box shows criteria (I3,I4).

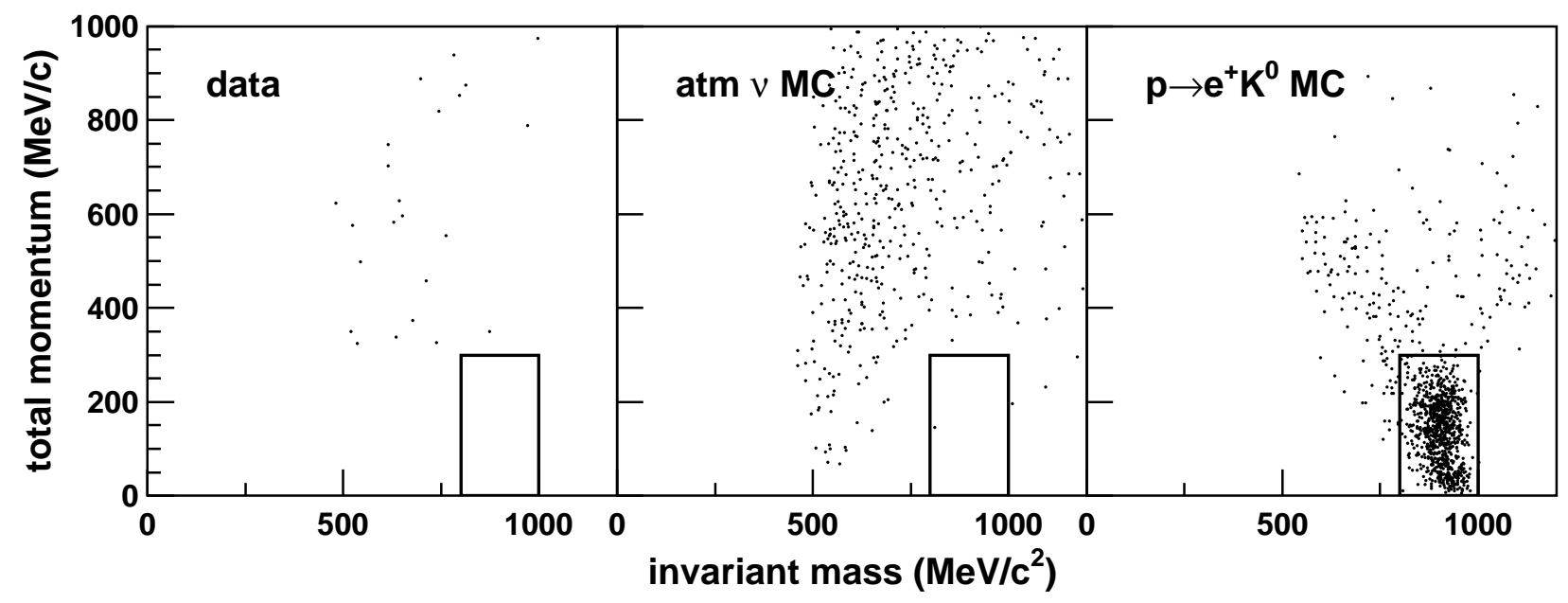

FIG. 13: The distributions of the total momentum versus invariant mass for events that satisfy criteria (J1-J3). Each figure shows: (left) data, (middle) atmospheric neutrino MC and (right) $p \rightarrow e^{+} K^{0} \mathrm{MC}$. The box shows criteria (J4,J5). 
uncertainty comes from the uncertainty of de-excitation gamma-ray emission probabilities. The total contributions in the $p \rightarrow \bar{\nu} K^{+}$, mono-energetic and $K^{+} \rightarrow \pi^{+} \pi^{0}$ search are estimated to be $2.5 \%$ and $8.8 \%$, respectively. Most of the uncertainties in the $K^{+} \rightarrow \pi^{+} \pi^{0}$ search come from the imperfect knowledge of charged pion-nucleon cross section in water and light scattering in water, which are estimated to be $4.8 \%$ and $6.7 \%$, respectively. In the $n \rightarrow \bar{\nu} K^{0}$ search, the total contributions are estimated to be $16 \%$ and $14 \%$ for the $K_{S}^{0} \rightarrow \pi^{0} \pi^{0}$ and $K_{S}^{0} \rightarrow \pi^{+} \pi^{-}$ search, respectively. The main source of uncertainty comes from the uncertainty in light scattering in water. In all methods of the $p \rightarrow \mu^{+} K^{0}$ and $p \rightarrow e^{+} K^{0}$ searches, the total contributions are less than $20 \%$.

In the background estimation, we consider the following common systematic uncertainties in all searches: imperfect knowledge of atmospheric neutrino flux, neutrino cross sections, energy scale, and particle identification. Because we use the scaled MC, the absolute normalization error becomes smaller. However, the background uncertainty from the atmospheric neutrino flux normalization is conservatively estimated to be $20 \%$ [25]. The imperfect knowledge of the cross sections are considered to be $30 \%$ for quasi-elastic and elastic scattering, $30 \%$ for single meson production and $50 \%$ for deep inelastic scattering. From these sources of systematic uncertainty, the total contributions to the uncertainty of the background in the $n \rightarrow \bar{\nu} K^{0}, K_{S}^{0} \rightarrow \pi^{0} \pi^{0}$ and $K_{S}^{0} \rightarrow \pi^{+} \pi^{-}$search are estimated to be $44 \%$ and $41 \%$, respectively. The contributions in many other modes become more than $50 \%$, because background statistics of the atmospheric neutrino $\mathrm{MC}$ are poor. The uncertainties in all of the searches are summarized at Table

\section{F. Results}

In the absence of any significant nuleon decay signature, we interpret our results as lower limits of nucleon partial lifetime for each decay mode using the following method 31].

First, based on Bayes theorem, we calculate the nucleon decay probability, $P\left(\Gamma \mid n_{i}\right)$, as follows:

$$
P\left(\Gamma \mid n_{i}\right)=A \iiint \frac{e^{-\left(\Gamma \lambda_{i} \epsilon_{i}+b_{i}\right)}\left(\Gamma \lambda_{i} \epsilon_{i}+b_{i}\right)^{n_{i}}}{n_{i} !} P(\Gamma) P\left(\lambda_{i}\right) P\left(\epsilon_{i}\right) P\left(b_{i}\right) d \lambda_{i} d \epsilon_{i} d b_{i} .
$$

Here a Poisson distribution is assumed for the nucleon decay probability; $n_{i}$ is the number of candidate events in the $i$-th nucleon decay search; $\Gamma$ is the total decay rate; $\lambda_{i}$ is the corresponding detector exposure; $\epsilon_{i}$ is the detection efficiency including the meson branching ratio; and $b_{i}$ is the expected background. In our search, $\lambda_{i}$ is $3.05 \times 10^{34}$ proton-year for proton decay and $2.44 \times 10^{34}$ neutron-year for neutron decay. $P(\Gamma)$ is the decay rate probability density. We assume $P(\Gamma)$ as one for $\Gamma>0$ and otherwise zero. The uncertainties of detector exposure $\left(P\left(\lambda_{i}\right)\right)$, detection efficiency $\left(P\left(\epsilon_{i}\right)\right)$ and background $\left(P\left(b_{i}\right)\right)$ are expressed as follows:

$$
\begin{gathered}
P\left(\lambda_{i}\right)=\delta\left(\lambda_{i}-\lambda_{0, i}\right) \\
P\left(\epsilon_{i}\right)=e^{-\left(\epsilon_{i}-\epsilon_{0, i}\right)^{2} / 2 \sigma_{\epsilon, i}^{2}}\left(0 \leq \epsilon_{i} \leq 1, \text { otherwise } 0\right) \\
P\left(b_{i}\right)=\frac{1}{b_{i}} \int_{0}^{\infty} \frac{e^{-b^{\prime}}\left(b^{\prime}\right)^{n_{b, i}}}{n_{b, i} !} e^{-\frac{-\left(b_{i} C_{i}-b^{\prime}\right)^{2}}{2 \sigma_{b, i}^{2}}} d b^{\prime} \\
\left(0 \leq b_{i}, \text { otherwise } 0\right),
\end{gathered}
$$

where $\lambda_{0, i}$ is the estimated exposure, $\epsilon_{0, i}$ is the estimated detection efficiency, $\sigma_{\epsilon, i}$ is the estimated uncertainty in the detection efficiency, $n_{b, i}$ is the number of background events, $C_{i}$ is the $\mathrm{MC}$ oversampling factor, and $\sigma_{b, i}$ is the uncertainty in the background. Because the uncertainty in the exposure is small, a delta function is assumed for $P\left(\lambda_{i}\right)$. The lower limit of the nucleon decay rate, $\Gamma_{\text {limit }}$, is calculated using Equation 8 where $n$ is the number of searches for a decay mode. In our search, we calculate $90 \%$ confidence level (CL) limit, i.e., $C L=0.9$ as follows:

$$
C L=\frac{\int_{\Gamma=0}^{\Gamma_{\text {limit }}} \prod_{i=1}^{n} P\left(\Gamma \mid n_{i}\right) d \Gamma}{\int_{\Gamma=0}^{\infty} \prod_{i=1}^{n} P\left(\Gamma \mid n_{i}\right) d \Gamma} .
$$

The lower limit of partial nucleon lifetime, $\tau / B$, is now calculated by:

$$
\tau / B=\frac{1}{\Gamma_{\text {limit }}} \sum_{i=1}^{n}\left[\epsilon_{0, i} \cdot \lambda_{0, i}\right] .
$$

In the $p \rightarrow \bar{\nu} K^{+}$, prompt gamma-ray and $K^{+} \rightarrow \pi^{+} \pi^{0}$ search, the lower limit of the nucleon partial lifetime is found to be $1.0 \times 10^{33}$ years and $7.8 \times 10^{32}$ years at $90 \%$ $\mathrm{CL}$, respectively. Only for $p \rightarrow \bar{\nu} K^{+}$, mono-energetic muon search, we use a special method because it has a lot of background. We search for an excess in the signal region (B3) of the muon momentum distribution. After applying criteria (B1,B2,B7), the events are divided 
into three momentum bins; $p_{\mu}$ are $200-215 \mathrm{MeV} / c, 215$ $260 \mathrm{MeV} / c$ and 260-305 MeV/c. The numbers of events in each momentum bin $\left(n_{1}, n_{2}, n_{3}\right)$ are $76,181,185$ events, respectively. The expected numbers of neutrino
MC events in each momentum bin $\left(b_{1}, b_{2}, b_{3}\right)$ are 78,223 , 182 events, respectively. The nucleon decay probabilities, $P\left(\Gamma \mid n_{1}, n_{2}, n_{3}\right)$, is then calculated using Equation 10

$P\left(\Gamma \mid n_{1}, n_{2}, n_{3}\right)=A \iiint \prod_{i=1}^{3} \frac{e^{-\left(\Gamma \lambda_{i} \epsilon_{i}+b_{\text {shape }, i} * b\right)}\left(\Gamma \lambda_{i} \epsilon_{i}+b_{\text {shape }, i} * b\right)^{n_{i}}}{n_{i} !} P(\Gamma) P\left(\lambda_{i}\right) P\left(\epsilon_{i}\right) P(b) P\left(b_{\text {shape }, i}\right) d \lambda_{i} d \epsilon_{i} d b d b_{\text {shape }, i}$

where $i=1,2,3$, corresponds to $200-215 \mathrm{MeV} / c, 215-260$ $\mathrm{MeV} / c$ and $260-305 \mathrm{MeV} / c$, respectively; $P(b)$ is defined as one for $0<b$ and otherwise zero; $\epsilon_{1}, \epsilon_{2}$, and $\epsilon_{3}$ are estimated to be $0.25 \%, 34 \%$ and $1.3 \%$, respectively. The background shape, $b_{\text {shape }, i}$, is $b_{i}$ divided by $b_{2}$. The uncertainty function of the background shape $P\left(b_{\text {shape }, i}\right)$ is defined to be a Gaussian function for $i=1,3$ and a delta function for $i=2$. The uncertainties for $i=1,3$ are then estimated to be $7 \%$ and $8 \%$ from the $\mathrm{MC}$ model difference, respectively. From Equations 8 and 9 using $P\left(\Gamma \mid n_{1}, n_{2}, n_{3}\right)$ instead of $P\left(\Gamma \mid n_{i}\right)$, decay limit is calculated to be $6.4 \times 10^{32}$ years at $90 \% \mathrm{CL}$. Using the three methods, the combined lower limit on the partial lifetime of proton via $p \rightarrow \bar{\nu} K^{+}$is $2.3 \times 10^{33}$ years at $90 \% \mathrm{CL}$.

In the $n \rightarrow \bar{\nu} K^{0}$ analysis, although we observe more events than the expected background in the $K_{S}^{0} \rightarrow \pi^{+} \pi^{-}$ search, there is no excess in the $K_{S}^{0} \rightarrow \pi^{0} \pi^{0}$ search. Therefore we also set a nucleon decay lifetime limit in this article. Combining two methods, we obtain the lifetime limit of $1.3 \times 10^{32}$ years at $90 \%$ CL. In the $p \rightarrow \mu^{+} K^{0}$ and $p \rightarrow e^{+} K^{0}$ search, by combining three methods, the lifetime lower limits are set to be $1.3 \times 10^{33}$ years and $1.0 \times 10^{33}$ at $90 \% \mathrm{CL}$, respectively. The limits in all searches are summarized at Table I

\section{CONCLUSION}

We have searched for nucleon decay via $p \rightarrow \bar{\nu} K^{+}$, $n \rightarrow \bar{\nu} K^{0}, p \rightarrow \mu^{+} K^{0}$ and $p \rightarrow e^{+} K^{0}$ from an exposure of $92 \mathrm{kt} \cdot$ year. No significant excess above background is observed. The lower limits of the partial nucleon lifetime at $90 \%$ CL for each mode are $2.3 \times 10^{33}, 1.3 \times 10^{32}$, $1.3 \times 10^{33}$ and $1.0 \times 10^{33}$ years, respectively (See Table $\amalg$ ). From these results minimal SUSY SU(5) is fully excluded 9]. These results also give strong constraints on other SUSY GUT models [8, 11].

We gratefully acknowledge the cooperation of the Kamioka Mining and Smelting Company. The SuperKamiokande experiment has been built and operated from funding by the Japanese Ministry of Education, Culture, Sports, Science and Technology, the United States Department of Energy, and the U.S. National Science Foundation. Some of us have been supported by funds from the Korean Research Foundation (BK21) and the Korea Science and Engineering Foundation, the Polish Committee for Scientific Research (grant 1P03B08227), Japan Society for the Promotion of Science, and Research Corporation's Cottrell College Science Award.
[1] J.C.Pati and A.Salam, Phys. Rev. Lett.31, 661 (1973).

[2] H.Georgi and S.L.Glashow, Phys. Rev. Lett. 32, 438 (1974).

[3] C.McGrew et al., Phys. Rev. D59, 052004 (1999).

[4] K.Hirata et al., Phys. Lett. B220, 308 (1989).

[5] M.Shiozawa et al., Phys. Rev. Lett. 81, 3319 (1998).

[6] J.Wess and B.Zumino, Nucl. Phys. B70, 39 (1974).

[7] U.Amaldi et al., Phys. Lett. B260, 447 (1991).

[8] N.Sakai and T.Yanagida, Nucl. Phys. B197, 533 (1982); S.Weinberg, Phys. Rev. D26, 287 (1982); J.Ellis et al., Nucl. Phys. B202, 43. (1982); P.Nath et al., Phys. Rev. D32, 2348 (1985); P.Nath et al., Phys. Rev. D38, 1479
(1988); J.Hisano et al., Nucl. Phys. B402, 46 (1993); T.Goto and T.Nihei, Phys. Rev. D59, 115009 (1999).

[9] H.Murayama and A.Pierce, Phys. Rev. D65, 055009 (2002).

[10] Y. Fukuda et al., Phys. Rev. Lett. 81, 1562 (1998).

[11] K.S.Babu, J.C.Pati and F.Wilczek, Phys. Lett. B423, 337 (1998); J.C.Pati hep-ph/0204240.

[12] G.Battistoni et al., Phys. Lett. B133, 454 (1984); C.Berger et al., Nucl. Phys. B313, 509 (1989); C.Berger et al., Z. Phys. C50, 385 (1991); W.Allison et al., Phys. Lett. B427, 217 (1998).

[13] D.Wall et al., Phys. Rev. Lett. D61, 072004 (2000). 
TABLE I: Summary of nucleon decay search. The numbers in the parentheses are the systematic uncertainties (\%).

\begin{tabular}{|c|c|c|c|c|c|}
\hline mode & method & $\begin{array}{r}\text { efficiency } \\
(\%) \\
\end{array}$ & background & candidate & $\begin{array}{r}\text { lower limit } \\
\left(\times 10^{32} \text { years }\right) \\
\end{array}$ \\
\hline \multirow[t]{4}{*}{$p \rightarrow \bar{\nu} K^{+}$} & total & & & & 23 \\
\hline & prompt gamma-ray search & $8.6(20)$. & $0.7(59)$. & 0 & 10. \\
\hline & mono-energetic muon search & $35.6(2.5)$ & & - & 6.4 \\
\hline & $K^{+} \rightarrow \pi^{+} \pi^{0}$ search & $6.0(8.8)$ & $0.6(74)$. & 0 & 7.8 \\
\hline \multirow[t]{3}{*}{$n \rightarrow \bar{\nu} K^{0}$} & total & & & & 1.3 \\
\hline & $K_{S}^{0} \rightarrow \pi^{0} \pi^{0}$ & $6.9(16)$. & 19. (44.) & 14 & 1.3 \\
\hline & $K_{S}^{0} \rightarrow \pi^{+} \pi^{-}$ & $5.5(14)$. & 11. (41.) & 20 & 0.69 \\
\hline \multirow[t]{4}{*}{$p \rightarrow \mu^{+} K^{0}$} & total & & & & 13 \\
\hline & $K_{S}^{0} \rightarrow \pi^{0} \pi^{0}$ & 5.4 (11.) & 0.4 (78.) & 0 & 7.0 \\
\hline & $K_{S}^{0} \rightarrow \pi^{+} \pi^{-}$Method 1 & $7.0(9.5)$ & $3.2(41)$. & 3 & 4.4 \\
\hline & $K_{S}^{0} \rightarrow \pi^{+} \pi^{-}$Method 2 & $2.8(12)$. & $0.3(76)$. & 0 & 3.6 \\
\hline \multirow[t]{4}{*}{$p \rightarrow e^{+} K^{0}$} & total & & & & 10 \\
\hline & $K_{S}^{0} \rightarrow \pi^{0} \pi^{0}$ & $9.2(5.8)$ & 1.1 (62.) & 1 & 8.4 \\
\hline & $K_{S}^{0} \rightarrow \pi^{+} \pi^{-}$Method 1 & $7.9(12)$. & $3.6(50)$. & 5 & 3.5 \\
\hline & $K_{S}^{0} \rightarrow \pi^{+} \pi^{-}$Method 2 & 1.3 (19.) & 0.04 (146.) & 0 & 1.6 \\
\hline
\end{tabular}

[14] S. Fukuda et al., Nucl. Inst. and Meth. A501, 418 (2003).

[15] K.Nakamura et al., Nucl. Phys. A268, 381 (1976).

[16] T.Yamazaki and Y.Akaishi, Phys. Lett. B433, 1 (1999).

[17] H.Ejiri, Phys. Rev. C48, 1442 (1993).

[18] R.Woods and D.Saxon, Phys. Rev. 95, 577 (1954).

[19] J.Hyslop et al., Phys. Rev. D46, 961 (1992).

[20] R.Glasser et al., Phys. Rev. D15, 1200 (1977).

[21] CERN Program Library W5013 (1994).

[22] M.Nakahata et al., J. Phys. Soc. Jpn. 55, 3786 (1986); A.S.Clough et al., Nucl. Phys. B76 15 (1974).

[23] J.P.Albanese et al., Nucl. Phys. A350 301 (1980); C.H.Q.Ingram et al., Phys. Rev. C27 1578 (1983).

[24] T.A.Gabriel et al., IEEE Trans. Nucl. Sci. 36, 14 (1989).
[25] M.Honda et al., Phys. Rev. D52, 4985 (1995); M.Honda et al., Phys. Rev. D64, 053011 (2001).

[26] to be published.

[27] A.Bodek and U.K.Yang, hep-ex/0203009.

[28] D.Rein and L.M.Sehgal, Ann. of Phys. 133, 79 (1981); D.Rein, Z. Phys. C35, 43 (1987).

[29] Y.Hayato et al., Phys. Rev. Lett. 83, 1529 (1999).

[30] C.Carone et al. Phys. Rev. D53 6282 (1996).

[31] Particle Data Group, Review of Particle Physics, Section: Errors and confidence intervals - Bounded physical region, June 1996: R.M. Barnett et al., Phys. Rev. D54, 375 (1996). 